\title{
Práticas assistenciais de enfermagem na prevenção da pneumonia associada à ventilação mecânica: revisão integrativa
}

Habitualmente as pneumonias hospitalares se desenvolvem devido à aspiração de microrganismos que colonizam a orofaringe e o trato gastrointestinal superior os pacientes internados em UTI encontram-se fisicamente enfraquecidos em consequência do trauma ou processo patológico sofrido, dificultando suas defesas orgânicas. Identificar na literatura quais prevenções é necessário para evitar a Pneumonia Associada à Ventilação (PAV) em pacientes internados nas Unidades de Terapia Intensiva. Trata-se de uma revisão integrativa realizada nas bases de dados Scielo e CAPES com os descritores: cuidados de enfermagem, pneumonia associada à ventilação mecânica, higiene bucal, publicados no período de 2012 a 2020. Foram levantados cuidados de enfermagem como decúbito elevado entre 30-40; higienização oral com clorexidina 0,12\%; aspirações de vias aéreas; avaliação da pressão do cuff; cuidados com circuitos do ventilador. A elevação do decúbito do paciente esteve presente em todos os estudos levantados, assim como a higiene oral, aspiração e avaliação da sedação, os estudos elegíveis evidenciam que os dados se assemelham com os demonstrados pelos protocolos da ANVISA. As práticas de enfermagem na prevenção da Pneumonia Associada à Ventilação são de suma importância para uma formação contínua do profissional de enfermagem, sendo necessário incluir na rotina escalas de bundle, protocolos operacionais padrão (POP), sempre renovando e ampliando seus conhecimentos permanentes para entender o melhor cuidado aplicado a cada paciente.

Palavras-chave: Cuidados de Enfermagem; Pneumonia Associada à Ventilação Mecânica; Higiene Bucal.

\section{Nursing care in the prevention of ventilator-associated pneumonia: an integrative review}

Usually nosocomial pneumonias develop due to the aspiration of microorganisms that colonize the oropharynx and upper gastrointestinal tract, patients admitted to the ICU are physically weakened as a result of the trauma or pathological process suffered, making their organic defenses difficult. To identify in the literature which prevention is necessary to avoid Ventilator-Associated Pneumonia (VAP) in patients hospitalized in Intensive Care Units. This is an integrative review carried out in the Scielo and CAPES databases with the descriptors: nursing care, ventilator-associated pneumonia, oral hygiene, published from 2012 to 2020. Nursing care was identified as elevated decubitus between $30-40$; ; oral hygiene with $0.12 \%$ chlorhexidine; airway aspirations; cuff pressure assessment; care of ventilator circuits. The elevation of the patient's position was present in all studies surveyed, as well as oral hygiene, aspiration and evaluation of sedation, the eligible studies show that the data are similar to those demonstrated by the ANVISA protocols. Nursing practices in the prevention of VentilatorAssociated Pneumonia are of paramount importance for the continuous training of nursing professionals, and it is necessary to include bundle scales, standard operating protocols (POP) in the routine, always renewing and expanding their permanent knowledge e to understand the best care applied to each patient.

Keywords: Nursing Care; Mechanical Ventilation Associated Pneumonia; Oral hygiene.

Topic: Terapia Intensiva

Reviewed anonymously in the process of blind peer.

Carmel Fernanda Augusto Gonçalves iD Universidade de Sorocaba, Brasil http://lattes.cnpq.br/7937071239898564 http://orcid.org/0000-0003-0968-7147 carmel.faugusto@gmail.com

Diego Longo dos Santos (it)

Universidade de Sorocaba, Brasil http://lattes.cnpq.br/2131913367935867

http://orcid.org/0000-0002-9597-6190

diego.longo09@gmail.com

Leandro Aparecido de Souza (iD Universidade de Sorocaba, Brasil http://lattes.cnpq.br/6090315154831086 http://orcid.org/0000-0001-8828-9918 leandro.souza@prof.uniso.br

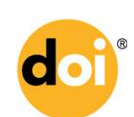

DOI: 10.6008/CBPC2236-9600.2021.003.0001
Received: 05/06/2021 Approved: 06/07/2021

Clayton Gonçalves de Almeida Universidade de Sorocaba, Brasil http://lattes.cnpq.br/6493791537446598 http://orcid.org/0000-0003-2959-3965 cgalmeida11@gmail.com

\section{Referencing this:}

GONÇALVES, C. F. A.; SANTOS, D. L.; SOUZA, L. A.; ALMEIDA, C. G.. Práticas assistenciais de enfermagem na prevenção da pneumonia associada à ventilação mecânica: revisão integrativa. Scire Salutis, v.11, n.3, p.1-5, 2021. DOI: http://doi.org/10.6008/CBPC2236$\underline{9600.2021 .003 .0001}$ 


\section{INTRODUÇÃO}

As Unidades de Terapia Intensiva (UTI) foram criadas a partir da necessidade de atendimento ao paciente em estado crítico exigindo uma assistência e observação contínua de profissionais de saúde. Os pacientes internados em UTI encontram-se fisicamente enfraquecidos em consequência do trauma ou processo patológico sofrido, dificultando suas defesas orgânicas (CRUZ et al., 2019).

Observa-se como sendo uma busca incansável, a tentativa pela manutenção da vida dos pacientes graves que precisam de monitorização e suporte contínuo para preservação de suas funções vitais, onde a maioria dos pacientes é submetida a procedimentos invasivos, como tubo orotraqueal, traqueostomia e ventilação mecânica, que dificultam a efetividade de seus mecanismos de defesa (CRUZ et al., 2019).

Habitualmente, as pneumonias hospitalares se desenvolvem devido à aspiração de microrganismos que colonizam a orofaringe e o trato gastrointestinal superior, provocadas por procedimentos de intubação e/ou extubação, ventilação mecânica prolongada, sondagens nasogástricas e infusão de dietas enterais, além do processamento inadequado de equipamentos de assistência ventilatória (ALECRIM et al., 2019).

A Pneumonia Associada à Ventilação Mecânica (PAV) é uma infecção pulmonar que se desenvolve em uma pessoa que está sob uso do ventilador. Pode ocorrer uma infecção quando entram em contato com o tubo e nos pulmões do paciente. Ela surge após 48-72 horas da intubação endotraqueal e instituição da ventilação mecânica invasiva (GONÇALVES, 2019)

A Ventilação Mecânica Invasiva (VMI) é um meio de suporte à vida utilizado em UTI, consistindo num método que assiste ou substitui à respiração espontânea do doente por ação de um ventilador, que é conectado ao mesmo por uma via artificial, seja tubo orotraqueal ou traqueostomia (GONÇALVES, 2019; ORLANDINI et al., 2012).

Sendo necessário ressaltar, que a higiene bucal, não só ajuda a manter o estado sadio da boca, dos dentes, das gengivas e lábios, assim como atua no fator de prevenção nas infecções do aparelho respiratório causado por microaspirações. Assim, promover o cuidado de enfermagem com excelência está intimamente relacionado com a redução das infecções hospitalares e relacionado à colonização da orofaringe por patógenos, principalmente associado à ventilação mecânica em pacientes internados em UTI (ORLANDINI et al., 2012; BARROS, 2019).

\section{METODOLOGIA}

Este estudo trata-se de uma revisão integrativa da literatura e para a seleção dos artigos, foi realizada a busca no portal da Biblioteca Virtual em Saúde (BVS). As bases de dados utilizadas foram Scientific Electronic Library Online (Scielo), Coordenação de Aperfeiçoamento de Pessoal de Nível Superior (CAPES). Foi utilizado filtros para artigos publicados entre 2012 a 2020, no idioma português e inglês, com os seguintes descritores: cuidados de enfermagem; pneumonia associada à ventilação mecânica; higiene bucal. Os critérios utilizados para inclusão foram: artigos científicos, teses e dissertações. A busca nas bases de dados se encontra sistematizada no fluxograma (figura 1). 


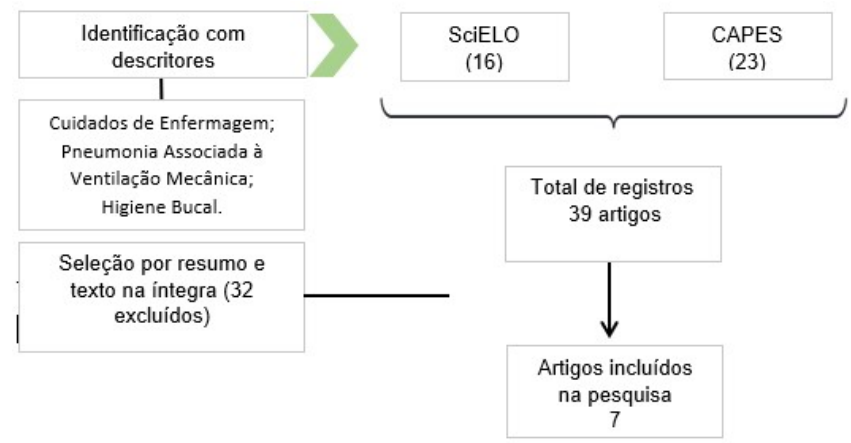

Figura 1: Fluxograma de seleção dos artigos por base de dados

\section{RESULTADOS E DISCUSSÃO}

O Quadro abaixo representa a síntese dos estudos identificados e incluídos na revisão integrativa.

Quadro 1: Caracterização dos estudos incluídos na pesquisa.

\begin{tabular}{|c|c|c|c|c|}
\hline & Autor/ano & Tipo de pesquisa & Título & Objetivo \\
\hline 1 & $\begin{array}{l}\text { Cruz et al. } \\
2019\end{array}$ & $\begin{array}{l}\text { Artigo científico } \\
\text { longitudinal } \\
\text { descritivo }\end{array}$ & $\begin{array}{l}\text { Pneumonia associada à } \\
\text { ventilação mecânica } \\
\text { invasiva: cuidados de } \\
\text { enfermagem }\end{array}$ & $\begin{array}{l}\text { Identificar os procedimentos de enfermagem em doentes } \\
\text { submetidos à ventilação mecânica invasiva e o } \\
\text { desenvolvimento de pneumonia num serviço de medicina } \\
\text { intensiva }\end{array}$ \\
\hline 2 & $\begin{array}{l}\text { Alecrim et } \\
\text { al. } 2019\end{array}$ & $\begin{array}{l}\text { Artigo científico } \\
\text { de coorte } \\
\text { prospectivo }\end{array}$ & $\begin{array}{l}\text { Boas práticas na prevenção } \\
\text { de pneumonia associada à } \\
\text { ventilação mecânica }\end{array}$ & $\begin{array}{l}\text { Avaliar a adesão dos profis sionais de saúde a um conjunto } \\
\text { de boas práticas de prevenção de Pneumonia Associada à } \\
\text { Ventilação Mecânica, índice de conformidade às medidas } \\
\text { individuais e associação de características clínicas dos } \\
\text { pacientes e adesão ao conjunto de boas práticas com a } \\
\text { pneumonia. }\end{array}$ \\
\hline 3 & $\begin{array}{l}\text { Sachetti et } \\
\text { al. } 2019\end{array}$ & $\begin{array}{l}\text { Estudo de coorte } \\
\text { transversal }\end{array}$ & $\begin{array}{l}\text { Adesão às medidas de um } \\
\text { bundle para prevenção de } \\
\text { pneumonia associada à } \\
\text { ventilação mecânica }\end{array}$ & $\begin{array}{l}\text { Avaliar a adesão ao bundle de ventilação mecânica em uma } \\
\text { unidade de terapia intensiva, bem como o impacto dessa } \\
\text { adesão nas taxas de pneumonia associada à ventilação } \\
\text { mecânica. }\end{array}$ \\
\hline 4 & $\begin{array}{l}\text { Silva et al. } \\
2014\end{array}$ & $\begin{array}{l}\text { Pesquisa } \\
\text { descritiva }\end{array}$ & $\begin{array}{l}\text { Pneumonia associada à } \\
\text { ventilação mecânica: } \\
\text { discursos de profissionais } \\
\text { acerca da prevenção }\end{array}$ & $\begin{array}{l}\text { Identificar os cuidados que os profissionais de enfermagem } \\
\text { e fisioterapia de uma Unidade de Terapia Intensiva } \\
\text { conhecem e consideram importantes para prevenção da } \\
\text { Pneumonia Associada à Ventilação Mecânica (PAV) }\end{array}$ \\
\hline 5 & $\begin{array}{l}\text { Orlandini et } \\
\text { al. } 2012\end{array}$ & $\begin{array}{l}\text { Estudo } \\
\text { exploratório } \\
\text { descritivo }\end{array}$ & $\begin{array}{l}\text { Conhecimento da equipe de } \\
\text { enfermagem sobre higiene } \\
\text { oral em pacientes } \\
\text { criticamente enfermos }\end{array}$ & $\begin{array}{l}\text { Avaliar o conhecimento dos profissionais de enfermagem, } \\
\text { que realizam ou supervisionam os cuidados de higiene oral } \\
\text { em pacientes críticos e, secundariamente, verificar como } \\
\text { julgam o cuidado prestado }\end{array}$ \\
\hline 6 & $\begin{array}{l}\text { Gonçalves } \\
\text { et al. } 2012\end{array}$ & & $\begin{array}{l}\text { Eficácia de estratégias } \\
\text { educativas para ações } \\
\text { preventivas da pneumonia } \\
\text { associada à ventilação } \\
\text { mecânica }\end{array}$ & $\begin{array}{l}\text { Determinar a eficácia de estratégia educativa para melhorar } \\
\text { o desempenho da equipe de enfermagem na realização de } \\
\text { procedimentos preventivos da pneumonia associada à } \\
\text { ventilação mecânica. }\end{array}$ \\
\hline 7 & $\begin{array}{l}\text { Gonçalves } \\
\text { et al. } 2012\end{array}$ & $\begin{array}{l}\text { Estudo } \\
\text { transversal } \\
\text { observacional }\end{array}$ & $\begin{array}{l}\text { Ações de enfermagem na } \\
\text { profilaxia da pneumonia } \\
\text { associada à ventilação } \\
\text { mecânica }\end{array}$ & $\begin{array}{l}\text { Identificar as ações da equipe de enfermagem relacionadas } \\
\text { à profilaxia da pneumonia associada à ventilação mecânica } \\
\text { (PAV) }\end{array}$ \\
\hline
\end{tabular}

Dentre os artigos levantados, foram encontrados os seguintes cuidados de enfermagem na prevenção da PAV:

Quadro 2: Decúbito elevado entre 30-45은
1. Higiene Ora
2. Avaliação da sedação
3. Aspiração de vias aéreas
4. Pressão do cuff entre $20-30 \mathrm{mmHg}$
5. Cuidado com o circuito do ventilador 
A elevação do decúbito do paciente esteve presente em todos os estudos levantados. Artigo 1 traz como evidência, que dentre os cuidados de enfermagem citados, a elevação do decúbito maior que 30으 apresenta uma taxa de cumprimento de quase $99 \%$, reduzindo ao todo $88,9 \%$ da incidência de aspiração, prevenindo assim o desenvolvimento da PAV. Já o Artigo 2, demonstra que apesar da elevação do decúbito seja uma prática de fácil aplicação, estudos demonstram uma baixa adesão dos profissionais a essa técnica. Mas convergem-se em relação à diminuição das taxas de aspiração. Os dados se assemelham com os demonstrados pelo protocolo da ANVISA, que apesar da escassez de estudos direcionados a esse tema e o real impacto na diminuição da PAV e mortalidade, é uma prática aceita dentro dos protocolos de PAV.

A higienização oral com gluconato de clorexidina esteve presente na maioria dos artigos e demonstrou um importante aliado na prevenção da PAV. No Artigo 5, um estudo realizado em uma UTI de um hospital de São Paulo, evidenciou uma taxa de adesão de $77,8 \%$ na prática da higiene oral. Dados similares ao do Artigo 6, que frisa a limpeza oral com clorexidina, devido seu grande potencial contra microrganismos gram-positivos e gram-negativo. O protocolo da ANVISA, refere que o uso da clorexidina 0,12\% associada a outras práticas, parece ter um efeito positivo na prevenção da PAV.

Conforme o protocolo da ANVISA, tem como base extubação do paciente. A otimização diária dos fármacos sedativos, assim como a mudança nas doses, influencia no tempo de permanência da ventilação mecânica. Dados similares aos do Artigo 6, que demonstra que a níveis de sedação leve e a interrupção diária da sedação, está associada a maior sobrevida dos pacientes em UTI. Porém, é uma prática que deve ser bem fundamentada por contas dos riscos que a interrupção da sedação pode trazer, como auto extubação, ansiedade, dor, assincronia com o ventilador e dessaturação. 0 Artigo 2, evidencia que a interrupção diária da sedação deve ser avaliada na visita multidisciplinar, conforme escala de monitorização de vigilância apropriada do cliente entubado.

A presença do tubo endotraqueal favorece a colonização bacteriana e a aspiração de secreções contaminadas pela diminuição do reflexo de tosse. A aspiração foi um cuidado citado em quase todos os artigos. O Artigo 7 frisa a importância da aspiração como forma de prevenção da PAV, mas também relata a necessidade de se manter fiel às técnicas corretas, para não trazem prejuízos secundários aos clientes sob ventilação mecânica. Outro ponto levantado no Artigo 1, foi o decúbito do paciente durante o procedimento. A cabeceira deve estar entre 30-40 para evitar risco de broncoaspiração. Dados que conversam com o protocolo da ANVISA. Que orienta que cliente em ventilação mecânica acima de $48 \mathrm{~h}$ ou $72 \mathrm{~h}$ deve ser aspirados rotineiramente e que essas práticas reduzem a incidência de PAV, diminuição o tempo de ventilação e internação na UTI.

Conforme protocolo da ANVISA, a manutenção da pressão de cuff é essencial, pois uma pressão excessiva pode interferir na microcirculação traqueal, ocasionando uma isquemia e a baixa pressão por interferir na pressão positiva e facilitar a aspiração de secreção orofaríngeas para vias aéreas inferiores. Artigo 2 relata que essa prática é considerada como nível de evidência III, devido os resultados de estudos anteriores não ter demonstrado impacto no tempo de permanência na UTI e taxa de mortalidade. Mas, é uma prática aceita no protocolo de prevenção da PAV, por apresentar efeitos satisfatórios na duração da 
ventilação mecânica. Porém, este mesmo artigo demonstra que um alto nível de não adesão a essa prática, chegando 51,5\%. Dados similares aos do Artigo 5, que traz a mesma porcentagem de não adesão, porém, com intervenções educativas, a adesão a essa prática foi para $66,7 \%$.

De acordo com o protocolo da ANVISA, a troca do circuito deve ser feita quando há presença visível de sujidade ou mau funcionamento. Essa recomendação é considerada com nível de evidência I. 0 Artigo 7, traz não só apenas o cuidado com o circuito, mas com os todos os equipamentos que participam do processo da ventilação mecânica. O cuidado com o acúmulo de condensado nos circuitos, que podem ocasionar uma pneumonia no cliente sob ventilação. Os dados convergem-se com os do Artigo 6, que salienta a vigilância na formação dos condensados nos circuitos e que a troca rotineira não está indicada.

\section{CONCLUSÕES}

Os cuidados do enfermeiro na prevenção da PAV são de suma importância para uma formação contínua do profissional de enfermagem, sendo necessário incluir na rotina escalas de bundle, protocolos operacionais padrão (POP), sempre renovando e ampliando seus conhecimentos permanentes para entender o melhor cuidado aplicado a cada paciente. São indicadores de novos protocolos e estudos para melhor prestação de cuidados ao paciente, como identificar as ações da equipe de enfermagem relacionadas à profilaxia da PAV.

Os estudos demonstraram que há necessidade da presença do enfermeiro para liderar e aplicar novas medidas, cuja complexidade repercute em uma dinâmica diferenciada como trabalho assistencial e gerenciar o cuidado ao paciente crítico em UTIs.

\section{REFERÊNCIAS}

ALECRIM, R. X.; TAMINATO, M.; BELASCO, A. G. S.. Boas práticas na prevenção de pneumonia associada à ventilação mecânica. Acta Paul Enferm, v.32, n.1, p.11-70, 2019. DOI: https://doi.org/10.1590/1982-0194201900003

BARROS, F. R. B.. Adesão ao bundle de prevenção de pneumonia associada à ventilação mecânica. Rev Cuid, v.10, n.2, 2019. DOI: https://doi.org/10.15649/cuidarte.v10i2.746

BRASIL. Medidas de Prevenção de Infecção Relacionada à Assistência à Saúde. Brasília: ANVISA, 2017.

CRUZ, J. R. M.; MARTINS, M. D. S.. Pneumonia associada à ventilação mecânica invasiva: cuidados de enfermagem. Revista de Enfermagem Referência, v.4, n.20, 2019. DOI: http://dx.doi.org/10.12707/RIV18035

GONÇALVES, F. A. F.; BRASIL, V. V.; RIBEIRO, L. C. M.; TIPPLE, A. F. V.. Ações de enfermagem na profilaxia da pneumonia associada à ventilação mecânica. Acta paulista de enfermagem, v.25, n.1, p.101-107, 2012. DOI:

\section{https://doi.org/10.1590/S0103-21002012000800016}

GONÇALVES, F. A. F.; BRASIL, V. V.; RIBEIRO, L. C. M.; TIPPLE, A. F. V.. Eficácia de estratégias educativas para ações preventivas da pneumonia associada à ventilação mecânica. Esc Anna Nery, v.16, n.4, p.802-808. DOI: https://doi.org/10.1590/S1414-81452012000400023

ORLANDINI, G. M.; LAZZARI, C. M.. Conhecimento da equipe de enfermagem sobre higiene oral em pacientes criticamente enfermos. Ver Gaúcha Enferm, v.33, n.3, p.3441, 2012. DOI: https://doi.org/10.1590/S198314472012000300005

SILVA, S. G.; NASCIMENTO, E. R. P.; SALLES, R. K.. Pneumonia Associada à ventilação mecânica: discursos de profissionais acerca da prevenção. Escola Anna Nery, v.18, n.2, p.290295, 2014. DOI: https://doi.org/10.5935/14148145.20140042

A CBPC - Companhia Brasileira de Produção Científica (CNPJ: 11.221.422/0001-03) detém os direitos materiais desta publicação. Os direitos referem-se à publicação do trabalho em qualquer parte do mundo, incluindo os direitos às renovações, expansões e disseminações da contribuição, bem como outros direitos subsidiários. Todos os trabalhos publicados eletronicamente poderão posteriormente ser publicados em coletâneas impressas sob coordenação da Sustenere Publishing, da Companhia Brasileira de Produção Científica e seus parceiros autorizados. Os (as) autores (as) preservam os direitos autorais, mas não têm permissão para a publicação da contribuição em outro meio, impresso ou digital, em português ou em tradução. 